\title{
Evaluation of clinical target volume expansion required for involved site neck radiotherapy for lymphoma to account for the absence of a pre-chemotherapy PET-CT in the radiotherapy treatment position
}

David Bird ${ }^{1}$, Chirag Patel ${ }^{2}$, Andrew Scarsbrook ${ }^{2}$, Viv Cosgrove ${ }^{1}$, Emma Thomas $^{3}$, Di Gilson ${ }^{3}$, Robin Prestwich ${ }^{3}$

\footnotetext{
${ }^{1}$ Medical Physics and Engineering, Bexley Wing, St. James's University Hospital, Leeds Teaching Hospitals Trust, Leeds, LS9 7TF, England.

2 Radiology and Nuclear Medicine, Bexley Wing, St. James's University Hospital, Leeds Teaching Hospitals Trust, Leeds, LS9 7TF, England.

${ }^{3}$ Clinical Oncology, Bexley Wing, St. James's University Hospital, Leeds Teaching Hospitals Trust, Leeds, LS9 7TF, England.
}

Corresponding Author:

Robin Prestwich

Email: Robin.Prestwich@nhs.net

Address: Level 4, St. James's Institute of Oncology, St. James's University Hospital, Beckett St., Leeds, LS9 7TF, UK

Tel: 00441132067838

Fax: 00441132067886

Total number of pages: 17

Total number of tables: 3 and 2 supplementary tables.

Total number of figures: 1

Running heading: Lymphoma ISRT CTV expansion

Key words: Lymphoma; radiotherapy; involved site; clinical target volume; PET-CT 


\section{Abstract}

\section{Background and purpose}

Involved site radiotherapy clinical target volume (CTV) for lymphoma requires an expansion to account for the absence of radiotherapy treatment-position pre-chemotherapy imaging, which is not widely implemented. This prospective imaging study aims to quantify CTV expansion required for neck radiotherapy.

\section{Materials and Methods}

10 patients from a prospective single centre imaging study underwent a pre-chemotherapy FDG-PET-CT in both the diagnostic and radiotherapy treatment position, and subsequently received neck radiotherapy post-chemotherapy. $\mathrm{CTV}_{\text {INRT }}$ and $\mathrm{CTV}_{\text {diagPET }}$ were delineated on the planning $\mathrm{CT}$, following co-registration of the radiotherapy position PET-CT and side-byside assessment of diagnostic PET-CT respectively.

\section{Results}

Intra-observer variability was limited, with delineation of CTV INRT highly reproducible and slightly lower for CTV diagPET (mean DICE 0.88 and 0.8 respectively). Superiorly, CTV $_{\text {diagPET }}$

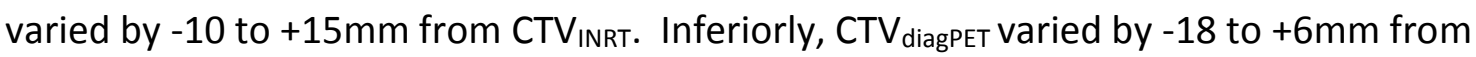
CTV INRT. $_{\text {IN }}$ Comparing CTV INRT $_{\text {Ind }}$ CTV $_{\text {diagPET }}$ in the axial plane, the mean DICE was 0.74. Mean sensitivity index was 0.75 (range $0.59-0.91$ ), showing that on average $75 \%$ of the CTV INRT was encompassed by the CTV $\mathrm{CiagPET}_{\text {. }}$

\section{Conclusions}

In the absence of treatment-position PET-CT, CTV expansion cranially and caudally by $10 \mathrm{~mm}$ and $18 \mathrm{~mm}$ respectively, along with generous contouring in the axial plane, was required to encompass pre-chemotherapy disease. 


\section{Introduction}

Radiotherapy continues to be widely used as part of curative treatment for Hodgkin (HL) and non-Hodgkin lymphoma (NHL). Late treatment-related complications have been highlighted, with second malignancy and cardiac toxicity identified as the most common cause of non-lymphoma deaths in HL survivors [1-3]. Radiotherapy dose to the head and neck region has been shown to increase stroke risk [4]. Multiple studies have demonstrated that complications are related to the irradiated volume $[5,6]$.

Modern lymphoma radiotherapy delivery aims to maintain local control rates whilst minimising radiation dose to normal tissues [7-10]. In 2006 Girinsky et al. [10] introduced the concept of involved nodal radiotherapy (INRT). INRT aims to treat only prior sites of nodal involvement and requires the acquisition of a pre-chemotherapy 2-[ ${ }^{18}$ Fluorine]-fluoro2-deoxy-D-glucose (FDG) positron emission tomography - computed tomography (PET-CT) in the radiotherapy treatment position with appropriate radiotherapy immobilisation devices with rigid co-registration to the post-chemotherapy planning CT. However, most centres have been unable to implement the requirement for INRT of a pre-chemotherapy PET-CT in the radiotherapy treatment position [11]. The International Lymphoma Radiation Oncology Group (ILROG) developed widely applicable guidelines for involved site radiotherapy (ISRT) $[7,8]$. In situations in which radiotherapy is delivered as part of combined modality treatment, the clinical target volume (CTV) is designed to encompass the extent of disease pre-chemotherapy, modified to anatomical boundaries, and expanded to account for any uncertainties in defining the pre-chemotherapy extent of disease, including the quality, position and accuracy of imaging $[7,8]$, depending upon clinical judgement [11]. INRT is regarded as ISRT when pre-chemotherapy PET-CT in the radiotherapy treatment position is available [7].

To date, there has been no data available to directly guide the expansion in CTV to account for the absence of optimal pre-chemotherapy imaging. In this prospective imaging study a series of patients underwent a pre-chemotherapy FDG PET-CT in both standard diagnostic and radiotherapy treatment positions. This study aims to evaluate the magnitude of uncertainty introduced into CTV delineation for nodal neck disease in the absence of a 
radiotherapy-position pre-chemotherapy $\mathrm{PET}-\mathrm{CT}$, in order to quantitatively inform on appropriate CTV expansion.

\section{Methods}

\section{Study outline}

This is a prospective single centre imaging study in patients with $\mathrm{HL}$ or high grade NHL with clinical \pm radiological (pre-PET-CT) stage I/II disease potentially suitable for treatment with sequential chemotherapy and radiotherapy (pending PET-CT staging outcome). The imaging protocol incorporates a pre-chemotherapy FDG-PET-CT acquired according to standard diagnostic protocols and, using the same FDG injection, acquisition of an additional FDGPET-CT scan with intravenous contrast with limited coverage of the head and neck region in the radiotherapy treatment position using radiotherapy mask immobilisation. Treatment was delivered according to institutional clinical protocols.

\section{Patient selection and recruitment}

Inclusion criteria were: age $\geq 18$ years old, male or female, histologically proven $\mathrm{HL}$ or high grade NHL, World Health Organisation (WHO) Performance status 0-2, Ann Arbor Stage I/II disease based upon clinical examination and any radiology investigations previously performed, residual disease in situ after biopsy, PET-CT staging not yet performed, clinical decision that sequential chemotherapy and radiotherapy will be the recommended treatment if stage $\mathrm{I} / \mathrm{II}$ disease is confirmed on subsequent PET-CT staging. This study was approved by the Research Ethics Committee. Trial registration: ISRCTN Registry: ISRCTN46587767.

A total of 19 patients were recruited between October 2013 and January 2016. All patients provided informed written consent. 12/19 patients subsequently underwent treatment with chemotherapy followed by radiotherapy. 10 of these patients underwent radiotherapy to the neck region and form the basis of this report.

\section{PET-CT Imaging}

A 5-point thermoplastic immobilisation mask was fabricated prior to PET-CT acquisition, with arms by sides; neck position was determined by the anatomical location of known 
disease and was either extended or neutral. FDG PET-CT imaging was performed on a 64section GE Discovery 690 PET-CT system (GE Healthcare, Amersham, UK) with a flat couch top. A standard diagnostic half-body PET-CT with arms up on a soft head support was performed 60 minutes following a 400 MBq injection of FDG intravenously. A dedicated PET$\mathrm{CT}$ of the head and neck region was then acquired with the immobilization mask in place with a radiotherapy head rest , arms down, (3-4 bed positions, 2 minutes per bed position) from skull vertex to carina. The CT component of the head and neck acquisition was obtained after a 25 second delay following a bolus of $100 \mathrm{ml}$ of iodinated contrast (Niopam 300, Bracco Ltd, High Wycombe, UK) injected at 3ml/s using the following settings; $120 \mathrm{kV}$, variable $\mathrm{mA}(\min 10, \max 600$, noise index 12.2 ), tube rotation $0.5 \mathrm{~s}$ per rotation, pitch 0.969 with a $2.5 \mathrm{~mm}$ slice reconstruction.

\section{Radiotherapy CT planning scan}

For patients who were subsequently treated with radiotherapy following chemotherapy the thermoplastic mask fabricated for the pre-chemotherapy PET-CT scan was fitted to assess whether the fit remained optimal. If this was not the case, a new thermoplastic mask was made attempting to maintain a similar neck position. The CT planning scan was acquired with intravenous contrast and 2 or $3 \mathrm{~mm}$ slice thickness (dependent upon institutional protocols at the time).

\section{CTV contouring}

Contouring was performed by radiation oncologists in a single centre specialising in the treatment of lymphoma, with access to clinical history and findings of clinical examination. When contouring using side-by-side assessment the clinician was blinded to the treatment position PET-CT. To minimise potential for recall, a minimum two week interval was mandated prior to generating contours for each individual patient using different methods.

\section{Contouring using co-registration of PET-CT acquired in radiotherapy position to planning}

CT scan

Contouring was performed according to the principles of the ILROG guidelines $[7,8]$. The contrast-enhanced pre-chemotherapy PET-CT acquired in the radiotherapy position was used to manually contour a gross tumour volume (GTV) based on the morphology of the CT and a GTV based upon the PET images, using predefined window and colour settings (SUV 
scale $0-7$, volcano colour scale for overlayed images). Rigid registration over the whole image was undertaken using Mirada RTx v1.4 software (Mirada Medical, Oxford, UK). All registrations were assessed for clinical suitability by a radiation oncologist. The postchemotherapy CTV (CTV INRT) was contoured, taking into account the co-registered prechemotherapy GTV and changes in lymphoma volume and anatomical position, whilst accounting for anatomical boundaries.

\section{Contouring using side-by-side assessment of PET-CT acquired in diagnostic position}

This simulates a situation in which optimal pre-chemotherapy PET-CT imaging in the treatment position is not available (CTV diagPET$_{\text {. }}$. A post-chemotherapy CTV aiming to encompass initially involved lymphoma tissue was contoured using the pre-chemotherapy diagnostic position PET-CT by side-by-side assessment, taking into account changes in lymphoma volume and anatomical changes, whilst accounting for anatomical boundaries. To allow quantification of the 'errors' introduced by contouring without optimal coregistered imaging, no additional CTV expansion was undertaken.

\section{Data analysis}

\section{Assessment of superior and inferior CTV extent}

Distance between the superior slices of the CTV INRT $_{\text {and }}$ CTV $V_{\text {diagPET }}$ was recorded. Distance was similarly recorded for inferior slices.

\section{Positional analysis}

Positional metrics were used to compare CTVs in the axial plane. The most superior and inferior overlapping slices of the CTVs (CTV INRT and CTV diagPET) were defined as the limits of the volume, excluding differences in the superior-inferior CTV length from influencing positional metrics. Positional metrics were also used to assess the intra-observer CTV variability, where all CTV delineations ( $\mathrm{CTV}_{\text {INRT }}$ or $\mathrm{CTV}_{\text {diagPET }}$ ) were compared to one another.

Five positional metrics were calculated using ImSimQA software (v3.1.5, OSL, Shrewsbury, UK): Mean distance to conformity (MDC); Centre of gravity distance (CGD); DICE index; conformality index $(\mathrm{Cl})$; sensitivity index (Se. Idx). The $\mathrm{Cl}$ and DICE index both produce output values between 0 and 1 (using different calculation methods), where 0 represents 
two contours with no overlap and 1 represents two contours that are perfectly overlapping (values are inherently $<1$ if contours are of differing volumes even if one encompasses the other) [12]. The Se. Idx calculates the overlapping volume between two contours as a percentage of the volume of one of the two contours, for the CTV INRT and CTV diagPET axial plane comparison, the Se. Idx. calculated the overlap between CTV $\mathrm{diagPET}_{\text {and }} \mathrm{CTV}_{\text {INRT }}$ as a percentage of the volume of CTV INRT. CGD is the distance between the geometric centres of two contours [13]. MDC is the mean of the distances between contours averaged over all positions not within the overlapping contour [13].

\section{Statistics}

All statistical analysis was performed using Matlab2013b (MATLAB and Statistics Toolbox Release 2013b, The MathWorks, Inc., Natick, Massachusetts, United States). Linear mixed effects models were used to determine the significance of the differences between CTV ${ }_{\text {INRT }}$ and CTV $_{\text {diagPET }}[14]$. Significances were determined for the difference in volume and for the following positional metrics: DICE, Se. Idx, CGD and MDC for the CTV INRT and CTV diagPET $_{\text {axial }}$ plane comparison and DICE, Se. Idx, CGD and MDC for the CTV INRT and CTV diagPET intraobserver comparisons. In all cases, the parameter (volume, DICE, Se. Idx, CGD or MDC) was a fixed effect variable and the patient was a random variable. Data population testing was performed using Q-Q plots to ensure the data was normally distributed. A significant $\rho$-value was considered to be $\rho<0.05$ [15]. 


\section{Results}

10 patients who entered the study had neck disease and received chemotherapy followed by radiotherapy to the neck region. Baseline characteristics, in addition to prechemotherapy GTV cranio-caudal length and volume are shown in Table 1. 7 patients had diffuse large B cell lymphoma and 3 had classical HL. All had nodal lymphoma with no extranodal sites. Mean pre-chemotherapy GTV was $64 \mathrm{mls}$. $8 / 10$ received 3 cycles of chemotherapy followed by radiotherapy. 2 patients with DLBCL received 6 cycles of chemotherapy followed by radiotherapy.

\section{Intra-observer variation in CTV generation}

Supplementary Table 1 summarises intra-observer variation in delineating CTV INRT and CTV $_{\text {diagPET }}$ based upon delineating 3 consecutive patients 3 times each. Based upon multiple positional metrics, CTV INRT was highly reproducible (mean DICE index 0.88 ) with minimal variability on the superior and inferior extent of the CTV; metrics for CTV $\mathrm{d}_{\text {diagPET }}$ showed higher intra-observer variability although reproducibility remained high (mean DICE index 0.80 ) and variability in the superior and inferior extent of the CTV was very limited.

\section{Intra-observer variation in CTV generation}

Supplementary Table 2 shows inter-observer variation in delineating CTV INRT $_{\text {and }}$ CTV diagPET $_{\text {Ia }}$ based upon 3 observers delineating 3 consecutive patients. CTV INRT was more reproducible than CTV $_{\text {diagPET }}$ (mean DICE index 0.83 versus 0.71 ). Variation of the superior extent of CTV $_{\text {diagPET }}$ compared with CTV INRT was -8 to $+6 \mathrm{~mm}$. Variation of the inferior extent of $\mathrm{CTV}_{\text {diagPET }}$ compared with $\mathrm{CTV}_{\text {INRT }}$ was -8 to $+8 \mathrm{~mm}$.

\section{Comparison of CTV INRT and CTV $\mathrm{CdiagPET}_{\text {: }}$ superior and inferior extent}

Figure 1A-D illustrates an example of comparison of CTV INRT and CTV diagPET $_{\text {showing }}$ diagnostic imaging (1A-B) representative examples of the superior and inferior extent of the CTVs in the coronal (1C) and sagittal (1D) planes. Table 2 summarises CTV volumes and the

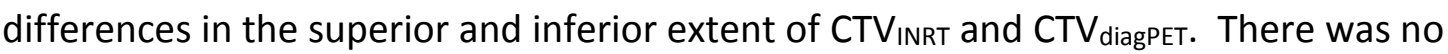
significant difference in volume between CTV INRT $_{\text {INd }}$ anV $V_{\text {diagPET }}(p=0.8)$. For the superior CTV

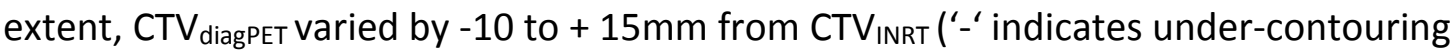
compared with CTV $\mathrm{INRT}_{\text {, and ' } ~}+$ ' indicates over-contouring), with a mean difference of $+0.5 \mathrm{~mm}$. For the inferior CTV extent, CTV diagPET Varied by -18 to $+6 \mathrm{~mm}$ compared with 
CTV INRT, with a mean difference of $-3.8 \mathrm{~mm}$. Based upon these data, an additional expansion of $10 \mathrm{~mm}$ superiorly and $18 \mathrm{~mm}$ inferiorly on the $\mathrm{CTV}_{\text {diagPET }}$ is required to encompass all instances of the length of the CTV INRT.

\section{Comparison of CTV INRT and $\mathrm{CTV}_{\text {diagPET }}$ : in axial plane}

Figure $1 \mathrm{E}-\mathrm{F}$ provides examples of the variation between $\mathrm{CTV}_{\text {INRT }}$ and $\mathrm{CTV}_{\text {diagPET }}$ in the axial plane. The positional metrics comparing $\mathrm{CTV}_{\text {INRT }}$ and $\mathrm{CTV}_{\text {diagPET }}$ in the axial plane are shown in Table 3. The mean DICE was 0.74. Mean Se.Idx was 0.75 (range 0.59-0.91), showing that on average $75 \%$ (range $59-91 \%$ ) of the $\mathrm{CTV}_{\text {INRT }}$ was covered by the CTV was $6.18 \mathrm{~mm}$ (range 2.68-10.02). MDC encompasses both under- and overlap between the $\mathrm{CTV}_{\text {INRT }}$ and $\mathrm{CTV}_{\text {diagPET. }}$ Mean and maximum MDC under-coverage (where the undercoverage region was defined as the region within CTV $_{\text {INRT }}$ which did not overlap with $\mathrm{CTV}_{\text {diagPET}}$ ) were $2.6 \mathrm{~mm}$ (range 1-4.8) and $7.4 \mathrm{~mm}$ (range 1.5-14.3) respectively. Mean and maximum MDC over-coverage (where the over-coverage region was defined as the region within CTV $_{\text {diagPET }}$ which did not overlap with CTV $_{\text {INRT }}$ ) were $3.6 \mathrm{~mm}$ (range 1.2-6.7) and $10 \mathrm{~mm}$

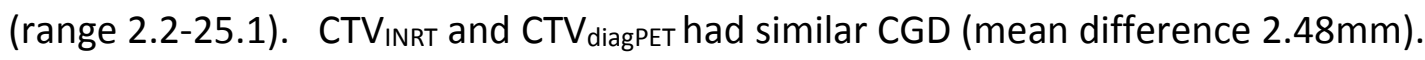




\section{Discussion}

FDG PET-CT is a routine staging investigation for lymphoma [16] and identifies sites of disease which need to be included within a CTV which are not identified upon CT imaging [17]. Use of PET-CT is essential to minimise the irradiation volume with ISRT whilst maintaining local control $[7,8,10]$. The concept of ISRT includes an expansion of the CTV to account for uncertainties in imaging including differences in the position in which the prechemotherapy PET-CT is acquired compared with the planning $\mathrm{CT}[7,8]$; this expansion is based upon clinical judgement [11] rather than a quantified process. In parallel with the development of the ILROG ISRT guidelines $[7,8]$, the UK National Cancer Research Institute Lymphoma Radiotherapy Group [9] developed ISRT guidelines for nodal lymphoma based upon clinical consensus, which specified an additional contoured $1.5 \mathrm{~cm}$ expansion craniocaudally in the direction of lymphatic spread from the pre-chemotherapy extent of nodal disease with no additional expansion specified in the axial plane.

In this prospective imaging study the acquisition of PET-CT with radiotherapy immobilisation allows generation of INRT CTVs used as a 'gold standard' with which to compare any 'inaccuracy' introduced by contouring using a diagnostic PET-CT used side-by-side with the planning PET-CT. Evaluation of intra-observer variability demonstrated that contouring the $\mathrm{CTV}_{\text {INRT }}$ using co-registered radiotherapy-position PET-CT is a highly reproducible step (mean DICE 0.88). As expected, this variability was slightly higher for CTV $_{\text {diagPET, although the }}$ positional metrics still showed limited variability in this step (mean DICE 0.80). Similarly, there was minimal variability in determination of the superior and inferior extent of the CTV for both $\mathrm{CTV}_{\text {INRT }}$ and $\mathrm{CTV}_{\text {diagPET }}$ when considered separately. Similar results were obtained when evaluating inter-observer variability, although as would be expected this variation was slightly higher (mean DICE 0.83 for CTV $_{\text {INRT }}$ and 0.71 for CTV $_{\text {diagPET, with superior and inferior }}$ variation falling within that for a single observer for all 10 patients). Based upon these findings, subsequent comparisons between $\mathrm{CTV}_{\text {INRT }}$ and $\mathrm{CTV}_{\text {diagPET }}$ are likely to be predominantly accounted for by differences introduced by the use of directly co-registered treatment-position PET-CT or side-by-side evaluation of diagnostic-position PET-CT, rather than intra-observer variability in contouring. 
Comparison of the cranio-caudal extent of CTV INRT and CTV diagPET $_{\text {demonstrated that for the }}$ superior and inferior CTV extent, the maximum distance of 'under-contouring' of the CTV diagPET was 10 and $18 \mathrm{~mm}$ respectively; mean differences were small $(0.4 \mathrm{~mm}$ superiorly and $3.8 \mathrm{~mm}$ inferiorly). The patient with $18 \mathrm{~mm}$ under-contouring at the inferior CTV extent had disease extending into the low neck. The more limited accuracy of defining the inferior CTV extent may relate to the greater variation in position in the lower neck between the pre-chemotherapy PET-CT in the diagnostic position (arms up) and radiotherapy-position. These data are broadly supportive of the proposal for ISRT of a contoured CTV expansion cranio-caudally of $15 \mathrm{~mm}$ suggested by the UK guidelines [9], although based upon these data an expansion of $10 \mathrm{~mm}$ is sufficient superiorly whilst an expansion of $18 \mathrm{~mm}$ may be required in the caudal part of the CTV.

Analysis of positional metrics in the axial plane demonstrated that differences between CTV $_{\text {INRT }}$ and $C T V_{\text {diagPET }}$ are greater than can be accounted for by intra-observer variation (mean DICE 0.74 ). The mean Se.Idx of 0.75 suggests that on average only $75 \%$ of the CTV INRT was encompassed in the axial plane by the CTV diagPET. Small differences in CGD show that differences in other positional metrics are not predominantly due to shifts of the whole CTV. The range of maximum MDC under-coverage was $1.5-14.3 \mathrm{~mm}$ (mean 7.4). This is despite modification of CTVs to anatomical boundaries. It is noticeable that although superior and inferior CTV expansions are recommended by the UK guidelines [9], no additional allowance is recommended in the axial plane. These data suggest that a generous approach to contouring ISRT CTVs in the axial plane, within anatomical boundaries, is required in the absence of treatment-position pre-chemotherapy PET-CT.

The data provided by this study provide a general idea of the magnitude of uncertainty in generating CTVs in the neck, and the potential for intra- and inter-observer variation are relevant factors. Although on the sub-analysis intra- and inter-observer variability were limited, it should be noted that the inter-observer analysis is based upon three clinicians at a single centre who are used to reviewing each other's contours. It needs to be considered that there is potential that this variability may be higher between differing centres. It is also critical that clinical judgement should be used when applying these data to individual cases, based upon the anatomical location of the disease in relation to easily identifiable 
landmarks and the quality/position of pre-chemotherapy imaging. For example, smaller margins may be appropriate when the target volume lies in proximity to easily identifiable anatomical landmarks, whilst larger margins may be appropriate when there is greater clinical uncertainty. In addition, in this study patients were treated with arms down with an immobilisation mask; in other circumstances, eg. involvement of the neck and mediastinum, it is possible to deliver treatment with arms up in a position similar to that adopted for the diagnostic PET-CT which may aid side-by-side comparison with diagnostic imaging but may require a larger margin to the PTV due to greater set up variability. This type of individualised approach to CTV generation reflects that recommended within the ILROG guidelines $[7,8]$. It should also be noted that these data cannot be used to guide CTV expansion for ISRT in other anatomical sites eg. mediastinum; easily identifiable anatomical structures within the neck is likely to lead to less uncertainty compared with other sites.

This analysis includes a limited number of patients with variable anatomical distribution and volume of pre-chemotherapy neck disease. Therefore, it is not possible to determine

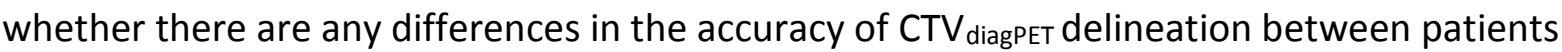
with large/small volume disease, upper/lower neck disease. There is no clear consensus regarding the optimal method for contouring GTV on PET-CT for multiple tumour types; methods can broadly include visual interpretation or methods of automatic segmentation $[18,19]$. It is clear for lymphoma that CT-derived GTV should be included as well as a PETderived GTV $[7,17]$. We utilised visual interpretation for this study with strictly controlled image viewing and windowing.

In summary, CTV expansion is required when contouring an ISRT CTV for neck lymphoma when a pre-chemotherapy PET-CT in the radiotherapy treatment position is not available. Based upon these data, additional CTV expansion cranio-caudally by $10 \mathrm{~mm}$ superiorly and up to $18 \mathrm{~mm}$ inferiorly may be necessary, although needs to be individualised depending upon the degree of clinical uncertainty with regard to position and proximity to anatomical landmarks. Contouring should also be generous in the axial plane, whilst respecting anatomical boundaries. 


\section{Acknowledgements}

We would like to acknowledge the assistance of Mrs Heather Hall in identifying suitable patients for the study.

\section{Role of funding source}

The study was funded by the The Leeds Teaching Hospitals Charitable Foundation.

\section{Conflicts of interest}

We have no conflicts of interest to declare. 


\section{References}

1. Aleman BM, van den Belt-Dusebout AW, Klokman WJ, Van't Veer MB, Bartelink H, van Leeuwen FE. Long-term cause-specific mortality of patients treated for Hodgkin's disease. J Clin Oncol 2003;21:3431-9.

2. $\mathrm{Ng} A K$, Bernardo MP, Weller $\mathrm{E}$, et al. Long-term survival and competing causes of death in patients with early-stage Hodgkin's disease treated at age 50 or younger. J Clin Oncol 2002;20:2101-8.

3. Schaapveld M, Aleman BM, van Eggermond AM, et al. Second Cancer Risk Up to 40 Years after Treatment for Hodgkin's Lymphoma. N Engl J Med 2015;373:2499-511.

4. Plummer C, Henderson RD, O'Sullivan JD, Read SJ. Ischemic stroke and transient ischemic attack after head and neck radiotherapy: a review. Stroke 2011;42:2410-8.

5. De Bruin ML, Sparidans J, van't Veer MB, et al. Breast cancer risk in female survivors of Hodgkin's lymphoma: lower risk after smaller radiation volumes. J Clin Oncol 2009;27:4239-46.

6. Franklin J, Pluetschow A, Paus $M$, et al. Second malignancy risk associated with treatment of Hodgkin's lymphoma: meta-analysis of the randomised trials. Ann Oncol 2006;17:1749-60.

7. Specht L, Yahalom J, Illidge T, et al. Modern radiation therapy for Hodgkin lymphoma: field and dose guidelines from the international lymphoma radiation oncology group (ILROG). Int J Radiat Oncol Biol Phys 2014;89:854-62.

8. Illidge T, Specht L, Yahalom J, et al. Modern radiation therapy for nodal non-Hodgkin lymphoma-target definition and dose guidelines from the International Lymphoma Radiation Oncology Group. Int J Radiat Oncol Biol Phys 2014;89:49-58.

9. Hoskin PJ, Diez P, Williams M, Lucraft H, Bayne M. Recommendations for the use of radiotherapy in nodal lymphoma. Clin Oncol (R Coll Radiol) 2013;25:49-58.

10. Girinsky $T$, van der Maazen $R$, Specht $L$, et al. Involved-node radiotherapy (INRT) in patients with early Hodgkin lymphoma: concepts and guidelines. Radiother Oncol 2006;79:270-7.

11. Hoppe BS, Hoppe RT. Expert radiation oncologist interpretations of involved-site radiation therapy guidelines in the management of Hodgkin lymphoma. Int J Radiat Oncol Biol Phys 2015;92:40-5.

12. Dice LR. Measures of the amount of ecologic association between species. . Ecology 1945;26:3:297-302.

13. Jena R, Kirkby NF, Burton KE, Hoole AC, Tan LT, Burnet NG. A novel algorithm for the morphometric assessment of radiotherapy treatment planning volumes. Br J Radiol 2010;83:44-51.

14. West BT. Linear mixed models: A practice guide using statistical software. 2nd Edition ed: CRC Press; 2015.

15. NIST/SEMATECH e-Handbook of Statistical Methods. (Accessed Accessed 09-12-14, at

16. Cheson BD, Fisher RI, Barrington SF, et al. Recommendations for initial evaluation, staging, and response assessment of Hodgkin and non-Hodgkin lymphoma: the Lugano classification. J Clin Oncol 2014;32:3059-68. 
17. Girinsky T, Ghalibafian M, Bonniaud G, et al. Is FDG-PET scan in patients with early stage Hodgkin lymphoma of any value in the implementation of the involved-node radiotherapy concept and dose painting? Radiother Oncol 2007;85:178-86.

18. Lee JA. Segmentation of positron emission tomography images: some recommendations for target delineation in radiation oncology. Radiother Oncol 2010;96:302-7.

19. Prestwich RJ, Sykes J, Carey B, Sen M, Dyker KE, Scarsbrook AF. Improving target definition for head and neck radiotherapy: a place for magnetic resonance imaging and 18fluoride fluorodeoxyglucose positron emission tomography? Clin Oncol (R Coll Radiol) 2012;24:577-89. 
Figure 1: Comparison of cranio-caudal length of $\mathrm{CTV}_{\text {INRT }}$ and $\mathrm{CTV}_{\text {diagPET }}$ in the coronal plane. Patient has stage I diffuse large B cell lymphoma treated with 3 cycles of chemotherapy followed by radiotherapy. A) represents prechemotherapy FDG PET-CT acquired in the radiotherapy treatment-position, B) represents routine diagnostic pre-chemotherapy PET-CT with arms up, C-F)

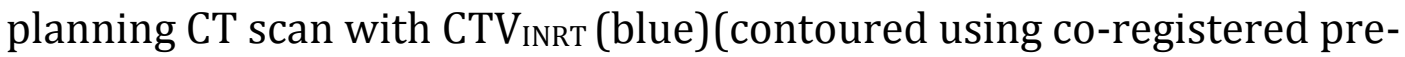
chemotherapy radiotherapy treatment-position PET-CT) and CTV diagPET (red) (contoured using side-by-side assessment of diagnostic PET-CT) in the coronal plane (C), in the sagittal plane (D), in the axial plane at the inferior extent of the CTVs (E), in the axial plane at the superior extent of the CTVs (F).

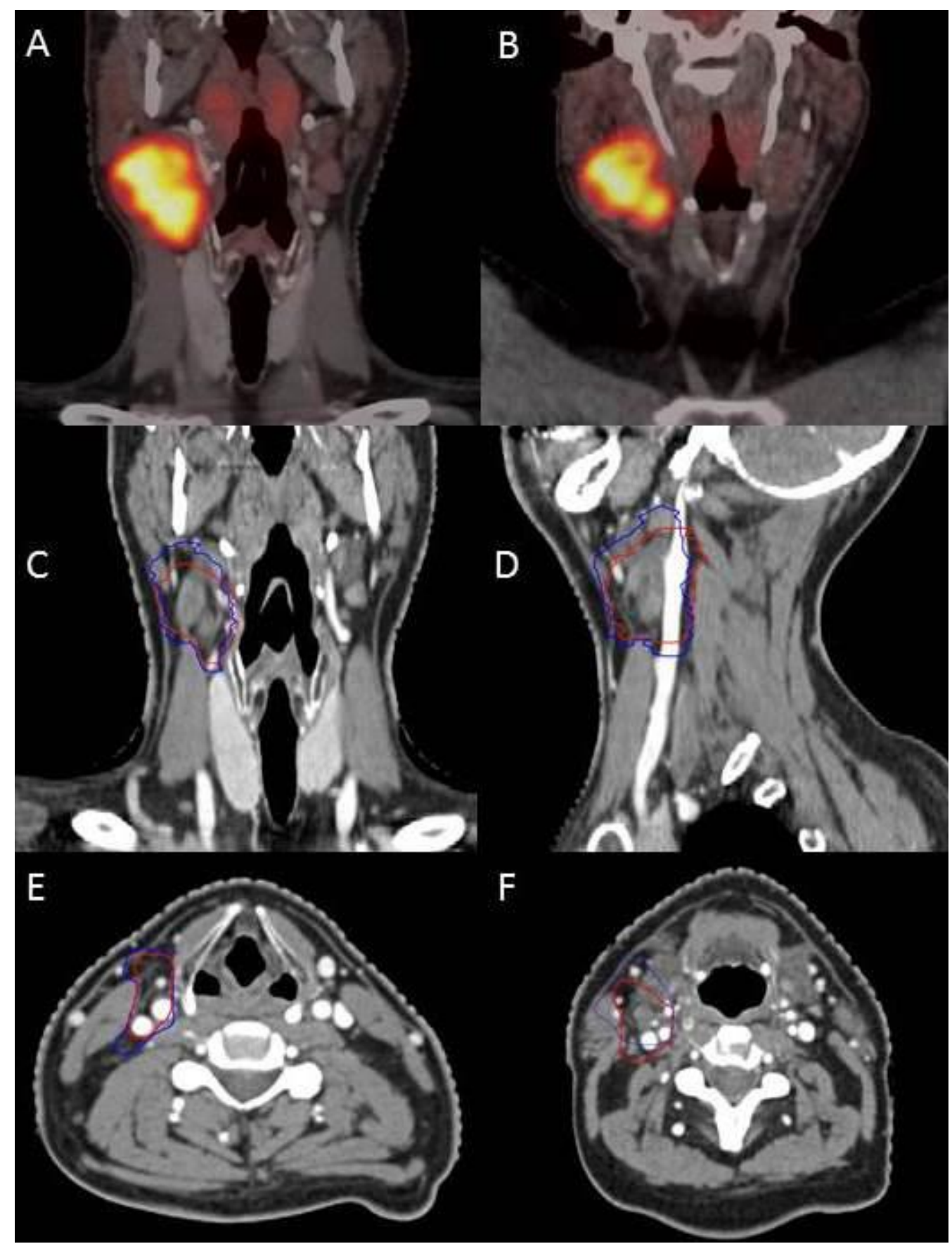


Table 1: Patient demographics, disease and treatment details

\begin{tabular}{|c|c|c|c|c|c|c|c|}
\hline Patient & $\begin{array}{l}\text { Lymphoma } \\
\text { type }\end{array}$ & Stage & RT Site & Age & Treatment & $\begin{array}{l}\text { Pre-chemotherapy } \\
\text { GTV/mls }\end{array}$ & $\begin{array}{l}\text { Pre-chemotherapy } \\
\text { GTV craniocaudal } \\
\text { length/mm* }\end{array}$ \\
\hline 1 & Hodgkin & la & Left neck & 29 & $3 \times A B V D+R T$ & 102 & 161 \\
\hline 2 & DLBLC & la & Left neck & 49 & $\begin{array}{l}3 \times \mathrm{RCHOP}+ \\
\mathrm{RT}\end{array}$ & 16 & 34 \\
\hline 3 & DLBCL & la & Right neck & 43 & $\begin{array}{l}3 \times R C H O P+ \\
\text { RT }\end{array}$ & 55 & 64 \\
\hline 4 & DLBCL & la & Left neck & 74 & $6 x \mathrm{RCHOP}+\mathrm{RT}$ & 77.8 & 64 \\
\hline 5 & DLBCL & la & Right neck & 69 & $\begin{array}{l}3 \times \mathrm{RCHOP}+ \\
\mathrm{RT}\end{array}$ & 4.2 & 27 \\
\hline 6 & DLBCL & la & Left & 81 & $\begin{array}{l}3 \times R C H O P+ \\
\text { RT }\end{array}$ & 21 & 70 \\
\hline 7 & Hodgkin & II & $\begin{array}{l}\text { Right } \\
\text { neck/upper } \\
\text { paratracheal }\end{array}$ & 70 & $3 \times A B V D+R T$ & 5 & 74 \\
\hline 8 & DBLCL & III & Left neck & 75 & $6 \times \mathrm{RCHOP}+\mathrm{RT}$ & 197 & 88 \\
\hline 9 & DBLCL & la & Right neck & 42 & $\begin{array}{l}3 \times \mathrm{RCHOP}+ \\
\text { RT }\end{array}$ & 6.6 & 141 \\
\hline 10 & Hodgkin & la & Right neck & 21 & $3 \times A B V D+R T$ & 64 & 92 \\
\hline Mean & & & & & & 64.2 & \\
\hline Median & & & & & & 59.8 & \\
\hline
\end{tabular}

*Defined on pre-chemotherapy PET-CT in radiotherapy-position

Table 2. $C T V_{\text {INRT }}$ and $C T V_{\text {diagPET }}$ volumes and their variation in superior and inferior extent

\begin{tabular}{|c|c|c|c|c|c|c|}
\hline & \multicolumn{3}{|l|}{ Volume } & \multirow{2}{*}{$\begin{array}{c}\text { CoG }(\mathrm{mm}) \\
\text { (Sup-Inf } \\
\text { direction) }\end{array}$} & \multicolumn{2}{|c|}{$\begin{array}{c}\text { Margins from } \mathrm{CTV}_{\text {INRT }} \text { to } \mathrm{CTV}_{\text {diagPET }} \\
(\mathrm{mm})\end{array}$} \\
\hline Patient & $\mathrm{CTV}_{\text {INRT }}$ & CTV & Difference (\%) & & Superior & Inferior \\
\hline 1 & 91.9 & 94.1 & +2.4 & 0.54 & 3 & 3 \\
\hline 2 & 11.5 & 10.2 & -11.3 & 0.41 & 0 & 0 \\
\hline 3 & 42.6 & 40.9 & -4.0 & 5.48 & -10 & -6 \\
\hline 4 & 15.5 & 14.1 & -9.0 & 7.27 & 0 & -14 \\
\hline 5 & 4.1 & 3.3 & -19.5 & 2.24 & -6 & -4 \\
\hline 6 & 16.7 & 19.8 & +18.6 & 3.70 & 15 & 0 \\
\hline 7 & 46.4 & 33.6 & -27.6 & 5.00 & 3 & -6 \\
\hline 8 & 86.7 & 95.6 & +10.3 & 2.46 & -6 & -4 \\
\hline 9 & 64.3 & 76.3 & +18.7 & 1.67 & 6 & 6 \\
\hline 10 & 42.2 & 38.3 & -9.2 & 9.29 & 0 & -18 \\
\hline Mean & 42.19 & 42.62 & +3.1 & 3.81 & 0.5 & -3.8 \\
\hline Stdev & 31.1 & 34.4 & +15.5 & 2.93 & 7.1 & 7.3 \\
\hline
\end{tabular}


Table 3. Positional metric comparison of $C_{T V} V_{\text {INRT }}$ and $C T V_{\text {diagPET }}$ in the axial plane

\begin{tabular}{|cccccc|}
\hline Patient & $\mathrm{Cl}$ & $\mathrm{MDC}(\mathrm{mm})$ & $\mathrm{CGD}(\mathrm{mm})$ & DICE & Se.Idx \\
\hline 1 & 0.56 & 6.23 & 2.24 & 0.73 & 0.74 \\
\hline 2 & 0.71 & 3.83 & 0.72 & 0.84 & 0.81 \\
\hline 3 & 0.49 & 10.02 & 6.58 & 0.7 & 0.72 \\
\hline 4 & 0.59 & 4.47 & 2.26 & 0.75 & 0.79 \\
\hline 5 & 0.68 & 3.15 & 2.03 & 0.83 & 0.91 \\
\hline 6 & 0.8 & 2.68 & 0.59 & 0.89 & 0.89 \\
\hline 7 & 0.48 & 7.42 & 2.55 & 0.64 & 0.56 \\
\hline 8 & 0.5 & 6.81 & 2.07 & 0.73 & 0.78 \\
\hline 9 & 0.51 & 7.18 & 2.94 & 0.66 & 0.7 \\
\hline 10 & 0.42 & 9.99 & 2.8 & 0.6 & 0.6 \\
\hline Mean & 0.57 & 6.18 & 2.48 & 0.74 & 0.75 \\
\hline Stdev & 0.12 & 2.63 & 1.64 & 0.09 & 0.11 \\
\hline
\end{tabular}

Supplementary Table 1. Intra-observer variation in the delineation of $\mathrm{CTV}_{\text {INRT }}$ and CTV diagPET

\begin{tabular}{|c|c|c|c|c|c|c|c|}
\hline & \multirow[b]{2}{*}{ Patient } & \multirow[b]{2}{*}{$\mathrm{Cl}$} & \multirow[b]{2}{*}{$\mathrm{MDC}(\mathrm{mm})$} & \multirow[b]{2}{*}{ CGD (mm) } & \multirow[b]{2}{*}{ DICE } & \multicolumn{2}{|c|}{$\begin{array}{l}\text { Superior and inferior } \\
\text { extent variation }(\mathrm{mm})\end{array}$} \\
\hline & & & & & & Superior & Inferior \\
\hline \multirow{4}{*}{$\mathrm{CTV}_{\text {INRT }}$} & 1 & 0.65 & 4.15 & 3.24 & 0.82 & 0 & 0 \\
\hline & 2 & 0.80 & 2.73 & 1.17 & 0.91 & 0 & 3 \\
\hline & 3 & 0.80 & 2.73 & 1.51 & 0.92 & 2 & 2 \\
\hline & Mean & 0.75 & 3.21 & 1.97 & 0.88 & 0.7 & 1.7 \\
\hline \multirow{4}{*}{ CTV $\mathrm{V}_{\text {diagPET }}$} & 1 & 0.61 & 6.07 & 3.36 & 0.79 & 6 & 3 \\
\hline & 2 & 0.57 & 4.07 & 1.25 & 0.8 & 3 & 6 \\
\hline & 3 & 0.60 & 5.24 & 2.70 & 0.8 & 4 & 0 \\
\hline & Mean & 0.60 & 5.13 & 2.44 & 0.8 & 4.3 & 3 \\
\hline \multicolumn{2}{|c|}{$\begin{array}{l}\text { P-value (CTV INRT Vs. } \\
\text { CTV }_{\text {diagPET }}\end{array}$} & $<0.001$ & $<0.001$ & 0.2 & $<0.001$ & - & - \\
\hline
\end{tabular}


Supplementary Table 2. Inter-observer variation in the delineation of CTV INRT and CTV diagPET

\begin{tabular}{|c|c|c|c|c|c|}
\hline & Patient & $\mathrm{Cl}$ & $\mathrm{MDC}(\mathrm{mm})$ & CGD (mm) & DICE \\
\hline \multirow{4}{*}{$\mathrm{CTV}_{\text {INRT }}$} & 1 & 0.67 & 3.68 & 2.24 & 0.85 \\
\hline & 2 & 0.65 & 3.41 & 1.22 & 0.86 \\
\hline & 3 & 0.62 & 4.48 & 3.32 & 0.78 \\
\hline & Mean & 0.65 & 3.86 & 2.26 & 0.83 \\
\hline \multirow{4}{*}{ CTV diagPET } & 1 & 0.41 & 7.47 & 7.33 & 0.64 \\
\hline & 2 & 0.57 & 4.47 & 2.21 & 0.80 \\
\hline & 3 & 0.48 & 8.04 & 6.35 & 0.69 \\
\hline & Mean & 0.49 & 6.66 & 5.30 & 0.71 \\
\hline \multicolumn{2}{|c|}{ P-value (CTV INRT VS. CTV diagPET) } & $<0.001$ & $<0.001$ & 0.02 & $<0.001$ \\
\hline
\end{tabular}

